\title{
Increased levels of MMP-3, MMP-9 and MPO represent predictors of in-stent restenosis, while increased levels of ADMA, LCAT, ApoE and ApoD predict bare metal stent patency
}

\author{
Leos Pleva ${ }^{a, b}$, Pavlina Kusnierovac,d, Pavlina Plevovace, Jana Zapletalova ${ }^{f}$, Michal Karpisek $^{\mathrm{g}}$, Lucie Faldynova ${ }^{c, e}$, \\ Petra Kovarovac,h, Pavel Kukla ${ }^{\mathrm{b}}$
}

\begin{abstract}
Aims. We sought to identify biochemical predictors that indicate susceptibility to in-stent restenosis (ISR) after coronary artery bare-metal stenting.

Methods. A total of 111 consecutive patients with post-percutaneous coronary intervention (PCI) in-stent restenosis of a target lesion within 12 months were matched for age, sex, vessel diameter, and diabetes with 111 controls without post-PCI ISR. Plasma or serum levels of biochemical markers were measured: matrix metalloproteinases (MMP) 2, 3, 9; myeloperoxidase (MPO); asymmetric dimethylarginine (ADMA); lipoprotein (a) (Lp[a]); apolipoproteins E and D (ApoE and D); and lecitin-cholesterol acyltransferase (LCAT). Multivariable logistic regression association tests were performed. Results. Increased plasma MMP-3 (OR: 1.013; 95\% Cl: 1.004-1.023; $P=0.005)$, MMP-9 (OR: 1.014; 95\% Cl: 1.008-1.020; $P<0.0001$ ) or MPO (OR: 1,$003 ; 95 \% \mathrm{Cl}: 1.001-1.005 ; P=0.002$ ) was significantly associated with increased risk of ISR. Increased levels of ADMA (OR: $0.212 ; 95 \% \mathrm{Cl}: 0.054-0.827 ; P=0.026)$, ApoE (OR: $0.924 ; 95 \% \mathrm{Cl}: 0.899-0.951 ; P<0.0001)$, ApoD (OR: $0.919 ; 95 \%$ Cl: $0.880-0.959 ; P=0.0001)$, or LCAT (OR: $0.927 ; 95 \%$ Cl: $0.902-0.952 ; P<0.0001$ ) was associated with risk reduction. No correlation was found between plasma MMP-2 or Lp (a) and ISR risk.

Conclusions. Increased levels of MMP-3, MMP-9, and MPO represent predictors of ISR after bare-metal stent implantation. In contrast, increased ADMA, LCAT, and Apo E and D indicate a decreased in-stent restenosis occurrence.
\end{abstract}

Key words: in-stent restenosis, matrix metalloproteinases, myeloperoxidase, asymmetric dimethylarginine, apolipoproteins E, apolipoproteins D, lecitin-cholesterol acyltransferase

Received: April 28, 2015; Accepted with revision: July 14, 2015; Available online: September 3, 2015

http://dx.doi.org/10.5507/bp.2015.037

${ }^{a}$ Department of Clinical Studies, Faculty of Medicine, University of Ostrava, Czech Republic

${ }^{b}$ Department of Cardiovascular Diseases, University Hospital Ostrava

'Department of Biomedical Sciencies, Faculty of Medicine, University of Ostrava

${ }^{d}$ Department of Laboratory Medicine, University Hospital Ostrava

${ }^{e}$ Department of Medical Genetics, University Hospital Ostrava

${ }^{f}$ Department of Medical Biophysics, Faculty of Medicine and Dentistry, Palacky University Olomouc

${ }^{9} R \& D$ Department, Biovendor Inc., Brno

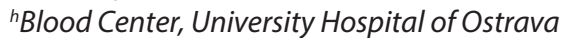

Corresponding author: Leos Pleva, e-mail: leos.pleva@volny.cz

\section{INTRODUCTION}

Coronary stent implantation has significantly improved percutaneous coronary intervention (PCI). It has enabled management of early complications of plane balloon angioplasty and by prevention of elastic recoil and constrictive remodeling, it decreases the frequency of restenosis after PCI. However, with these improvements has come a new complication: in-stent restenosis (ISR) arising from neointimal hyperplasia. The clinical incidence of ISR after bare-metal stent implantation is about 20-35\% (ref. ${ }^{1,2}$ ). The use of drug-eluting stents has led to a further decrease of ISR occurrence to $5-10 \%\left(\right.$ ref. ${ }^{1,2}$ ). However, this treatment requires long-term dual antiplatelet therapy, and is associated with the risk of late and very late stent thrombosis. The known predictors of ISR include patient-, vessel- and procedure-releated factors ${ }^{1,2}$. The increasing number of patients undergoing PCI has led to attempts to find further risk factors that would enable more targeted treatment.

We focused on possible additional biochemical predictors of ISR. Matrix metalloproteinases (MMPs) are proteolytic enzymes that degrade the extracellular matrix (ECM) and facilitate the proliferation and migration of endothelial and vascular smooth muscle cells (VSMC). We also considered plasma or serum levels of leucocyte myeloperoxidase (MPO), asymmetric dimethylarginine (ADMA), lipoprotein (a) (Lp[a]), apolipoproteins E and D (ApoE and D), and lecitin-cholesterol acyltransferase (LCAT), which may be involved not only in atherosclerosis but also in inflammation, possibly influencing proliferation and migration of smooth muscle cells (SMCs), which are key players in the development of ISR. 


\section{PATIENTS AND METHODS}

We included 111 consecutive patients treated at the cathlab of the University Hospital Ostrava, Czech Republic, between the years 2010 and 2013, with ISR within 12 month after implantation of a bare-metal stent.

The control group consisted of 111 matched patients treated at our cathlab with identical main demographic and clinical risk factors (sex, age, diabetes mellitus, implanted stent diameter $\pm 0.5 \mathrm{~mm}$ ) in whom ISR was excluded using multi-slice (MS-CT) coronarography 12 months after a bare-metal stent implantation.

\section{Selective coronarography}

Selective coronarography was performed in a Siemens Axiom device (Forchheim, Germany) using the standard method from the radial approach with $5 \mathrm{~F}$ diagnostic catheters and the contrast medium Iomeron 400. Quantitative coronary angiography was performed, and percent diameter stenosis (\%DS) was calculated. ISR was defined as a diameter stenosis $\geq 50 \%$ in the stented segment.

\section{Multi-slice CT coronarography}

The patients were examined in the Siemens Somatom Definition AS+ device (Forchheim, Germany), a singlesource CT scanner in a 128-slice configuration. The maximum intensity projection (MIP) reconstructions and automatic software Vessel analysis were used for evaluation of the lumen. Homogeneous enhancement (visually similar to the CT attenuation in the reference vessels) inside the stent lumen was considered to be normal or unrelated to ISR (ref. ${ }^{3}$ ).

\section{Blood samples and biochemical analysis}

Blood samles were collected 12 month after coronary intervention (rePCI for ISR or index PCI at controll group). Plasma samples from each patient were used for analysis of MMP-2, MMP-3, MMP-9, MPO, ApoE, ApoD and LCAT and serum samples for analysis of ADMA. Samples were centrifuged at $2500 \mathrm{~g}$ for $6 \mathrm{~min}$ at $4{ }^{\circ} \mathrm{C}$, aliquoted into 2 vials of $2 \mathrm{~mL}$, and stored at $-80{ }^{\circ} \mathrm{C}$ for 2-3 months.

Plasma MMP-2, MMP-3, MMP-9, ApoE, ApoD and LCAT and serum ADMA concentrations were determined by ELISA (enzyme-linked immunosorbent assay; Biovendor-Laboratorni Medicina, Brno, Czech Republic). The MMP-2, MMP-3, MMP-9, ApoE, ApoD and LCAT detection kits were labeled for research use, and used assays based on sandwich ELISA technology. The kit for ADMA detection was designed for in vitro use only, and was based on competitive ELISA technology.

The study protocol complied with the Declaration of Helsinki and was approved by the Ethics Committee of the University Hospital Ostrava, Czech Republic. Written informed consent was obtained from each participant.

\section{Statistical analysis}

Statistical analysis was performed using IBM SPSS Statistics version 22 (SPSS Inc., Chicago, IL, USA). Measured biochemical parameters were categorized according to reference values.

Continuous variables with non-normal distribution are presented as the median and range (minimum-maximum) and were compared using the non-parametric Mann-

Table 1. Clinical characteristics of patients (including matched controls) and angiographic parameters of coronary artery lesions.

\begin{tabular}{lccc}
\hline & In-stent restenosis & Controls & $P$ \\
\hline Age (years) & $67(60-73)$ & $67(61-71)$ & $0.732^{\mathrm{a}}$ \\
Male/Female & $66 / 45$ & $66 / 45$ & $1.000^{\mathrm{b}}$ \\
Diabetes mellitus (\%) & 33.33 & 33.33 & $1.000^{\mathrm{b}}$ \\
Renal insufficiency & 5.41 & 6.31 & $1.000^{\mathrm{b}}$ \\
BMI & $29(26.4-31.24)$ & $29.10(25.92-32.11)$ & $0.715^{\mathrm{a}}$ \\
Creat (umol/L) & $97.50(86-116)$ & $100(87.5-113)$ & $0.636^{\mathrm{a}}$ \\
glc (mmol/L) & $6.06(5.54-7.26)$ & $6.03(5.42-7.0)$ & $0.425^{\mathrm{a}}$ \\
Chol (mmol/L) & $4.27(3.73-4.90)$ & $4.62(3.97-5.63)$ & $0.016^{\mathrm{a}}$ \\
TG (mmol/L) & $1.63(1.19-2.19)$ & $1.58(1.14-2.28)$ & $0.823^{\mathrm{a}}$ \\
LDL (mmol/L) & $2.66(2.26-3.18)$ & $3.03(2.51-3.79)$ & $0.001^{\mathrm{a}}$ \\
HDL (mmol/L) & $1.24(1.02-1.46)$ & $1.21(1.01-1.40)$ & $0.521^{\mathrm{a}}$ \\
hs-CRP (mg/L) & $1.72(0.8-3.86)$ & $2.17(1.08-4.05)$ & $0.497^{\mathrm{a}}$ \\
NT-proBNP (ng/L) & $242(126.75-709.75)$ & $180(88-433.5)$ & $0.010^{\mathrm{a}}$ \\
Acute coronary syndromes (STEMI/NSTEMI) (\%) & 69.37 & 72.07 & $0.098^{\mathrm{b}}$ \\
Multi vessel dissease (\%) & 55.86 & 45.95 & $0.179^{\mathrm{b}}$ \\
B2/C lesions (\%) & 78.38 & 70.27 & $0.890^{\mathrm{b}}$ \\
Stent diameter(mm) & $3.0(3.0-3.5)$ & $3.25(3.0-3.5)$ & $0.218^{\mathrm{a}}$ \\
Stent lenght (mm) & $20(16-29)$ & $19(14.5-28)$ & $0.329^{\mathrm{a}}$ \\
\hline
\end{tabular}

Data are given as median (lower and higher quartiles); quantitative parameters are given in percents.

$P$ - significance of ${ }^{a}$ Mann-Whitney $\mathrm{U}$ test or ${ }^{\mathrm{b}}$ Fisher's exact test 
Whitney U test. Categorical variables are presented as counts and percentages and were compared by the chisquare test. The difference between measured biochemical parameters of the study and control groups was analyzed by the chi-square test (or the Fisher's exact test in the case of lower frequencies).

Multiple logistic regression was used to evaluate possible effects of other variables on the association observed between the levels of individual markers and ISR. A forward stepwise method was applied to identify the most significant predictive factors for ISR, with adjustment for diabetes mellitus and other possible confounding factors.

Receiver operating characteristic (ROC) analysis was used to determine the optimal cut-off values of biochemical parameters to predict in-stent restenosis. Normality of data was checked by Shapiro-Wilk test.

Odds ratios are expressed with $95 \%$ CIs. A $P$ value of less than 0.05 was considered statistically significant.

\section{RESULTS}

Clinical, biochemical and demographic characteristics of the study cohort are listed in Table 1. The patients with ISR and the control group did not show any significant differences with respect to the main demographic parameters (age, gender, body mass index) or clinical risk factors (diabetes mellitus). The groups exhibited similar extents of coronary disease (multi vessel disease, acute coronary syndromes) and similar lesion characteristics (complex lesion B2/C, and length and diameter $[ \pm 0.5$ $\mathrm{mm}$ ] of implanted stents). Furthermore, both groups exhibited mostly similar biochemical parameters (creatinine, glucose, triglycerides, HDL cholesterol, hsCRP). However, the ISR group exhibited significantly lower total cholesterol and LDL cholesterol, and significantly higher NT-pro-BNP, then the control group. Plasma levels of the individual biochemical parametrs measured in both groups are listed in Table 2.

Logistic regression analysis, conducted with adjustment for the prevalence of diabetes mellitus, revealed that an increase in MMP-3 (OR: 1.013; 95\% CI: 1.004-1.023; $P=0.005$ ), MMP-9 (OR: 1.014; 95\% CI: 1.008-1.020; $P<0.0001$ ), or MPO (OR: 1.003; 95\% CI: 1.001-1.005; $P=0.002)$ is significantly associated with increased occurrence of ISR and that an increase in ADMA (OR: 0.212; 95\% CI: 0.054-0.827; $P=0.026$ ), LCAT (OR: $0.927 ; 95 \%$ CI: 0.902-0.952; $P<0.0001$ ), ApoD (OR: 0.919; 95\% CI: 0.880-0.959; $P=0.0001$ ), or ApoE (OR: 0.924; 95\% CI: $0.899-0.951 ; P<0.0001)$ is significantly associated with decreased occurrence of ISR (Table 3 ).

Multivariate logistic regression analysis using a stepwise method to identify the most significant parameters for ISR prediction with adjustment for DM and individual parameters (hsCRP, Lpa, MMP-2, -3, -9, ADMA, LCAT, MPO, ApoD, ApoE) found that the most signifi-

Table 2. Plasma or serum ${ }^{a}$ levels of measured parametrs of patients (including matched controls).

\begin{tabular}{lcc}
\hline & In-stent restenosis & Controls \\
\hline Lp(a) $(\mathrm{g} / \mathrm{L})$ & $0.15(0.06-0.57)$ & $0.20(0.06-0.62)$ \\
MMP-2 $(\mathrm{ng} / \mathrm{mL})$ & $173.76(122.18-248.40)$ & $163.58(113.57-247.85)$ \\
MMP-3 $(\mathrm{ng} / \mathrm{mL})$ & $43.37(26.66-72.74)$ & $31.48(23.14-53.16)$ \\
MMP-9 $(\mathrm{ng} / \mathrm{mL})$ & $88.40(53.80-131.7)$ & $44.90(25.56-79.90)$ \\
ADMA $(\mathrm{mmol} / \mathrm{L})^{\mathrm{a}}$ & $0.36(0.21-0.53)$ & $0.46(0.35-0.57)$ \\
LCAT $(\mathrm{ug} / \mathrm{mL})$ & $39.11(34.17-44.22)$ & $52.43(41.85-59.66)$ \\
MPO $(\mathrm{ug} / \mathrm{mL})$ & $138.02(42.36-303.62)$ & $57.45(17.49-175.71)$ \\
Apo D $(\mathrm{ug} / \mathrm{mL})$ & $7.66(5.73-10.18)$ & $10.41(6.94-20.14)$ \\
Apo E $(\mathrm{ug} / \mathrm{mL})$ & $14.98(9.61-23.02)$ & $26.42(18.71-40.20)$ \\
\hline
\end{tabular}

Data are given as median (lower and higher quartiles)

Table 3. Logistic regression analysis (separetly for each parameter with adjustment for DM).

\begin{tabular}{lccc}
\hline Parameter & OR & $95 \%$ CI for OR & $P$ \\
\hline Hs-CRP & 0.791 & $0.404-1.550$ & 0.495 \\
Lp(a) & 1.000 & $0.956-1.046$ & 0.999 \\
MMP-2 & 1.001 & $0.999-1.004$ & 0.326 \\
MMP-3 & 1.013 & $1.004-1.023$ & 0.005 \\
MMP-9 & 1.014 & $1.008-1.020$ & $<0.0001$ \\
ADMA & 0.212 & $0.054-0.827$ & 0.026 \\
LCAT & 0.927 & $0.902-0.952$ & $<0.0001$ \\
MPO & 1.003 & $1.001-1.005$ & 0.002 \\
ApoD & 0.919 & $0.880-0.959$ & 0.0001 \\
ApoE & 0.924 & $0.899-0.951$ & $<0.0001$ \\
\hline
\end{tabular}

OR is associated with a single-unit increase in the value of the parameter 
cant predictive markers are ApoE (OR: 0.957; 95\% CI: 0.926-0.988; $P=0.008$ ) and LCAT (OR: $0.922 ; 95 \% \mathrm{CI}$ : 0.888-0.958; $P<0.0001)$. Increased abundance of ApoE or LCAT is associated with a significant reduction of ISR occurrence.

\section{Comparison with reference values for biochemical mark- ers in the healthy population}

Furthermore, we compared the levels of plasma of biochemical markers in ISR patients with the normal reference measurements of MMP-2, 3, 9 and ADMA in the healthy population (Appendix 1, 2) $\left(\right.$ ref. $\left.^{4}\right)$.

Logistic regression analysis was performed separately for each categorized parameter with adjustment for DM. A decrease in MMP-9 abundance below the lower limit of the reference range is associated with a significant reduction in the occurrence of ISR (OR: 0.265; 95\% CI: $0.121-0.582 ; P=0.001)$. Conversely, an increase in MMP9 above the upper limit of the reference range is associated with an increase in the occurrence of ISR (OR: 2.685; 95\% CI: 1.344-5.366; $P=0.005$ ). Increased MMP-3 above the upper limit of the reference range is associated with increased ISR occurrence (OR: 2.502; 95\% CI: 1.441-4.344; $P=0.001)$. Decreased ADMA below the lower limit of the reference range is also associated with ISR occurrence increase (OR: 2.115; 95\% CI: $1.105-4.050 ; P=0.024$ ).

We also performed multivariate logistic regression analysis using a stepwise method to choose the most significant categorized parameters predictive of ISR, with adjustment for DM and individual categorized parameters. The analysis indicated that most significant parameters with respect to prediction of ISR are MMP-9 (OR: 0.322; 95\% CI: 0.122-0.854; $P=0.023$ ), LCAT (OR: 0.927; 95\% CI: $0.892-0.964 ; P<0.0001$ ), and ApoE (OR: 0.967; 95\% CI: $0.935-0.999 ; P=0.048)$. A decrease in MMP-9 below the lower limit of the reference range is associated with a reduction in the occurrence of ISR. An increase in LCAT or ApoE is also associated with a reduction in the occurrence of ISR.

\section{Determination of appropriate cut-off values for ISR prediction}

Based on receiver operating characteristic (ROC) analysis, the plasma abundance of MMP-9, LCAT, and ApoE may be considered the most suitable parameters for use in ISR risk prediction (Appendix 3). The following cut-off values for prediction of ISR were determined: MMP-9 $\geq 64.8 \mathrm{ng} / \mathrm{mL}$; LCAT $\leq 42.39 \mathrm{ug} / \mathrm{mL}$ and ApoE $\leq 21.2 \mathrm{ug} / \mathrm{mL}$; with sensitivity and specificity of $65.8 \%$ and $65.8 \% ; 69.4 \%$ and $72.0 \% ; 67.70 \%$ and $69.20 \%$, respectively (Table 4A). Statistical analysis of parameter combinations of LCAT+ApoE and ApoE+ApoD indexes increased the sensitivity and specificity of ISR prediction to $75.5-77.0 \%$ and $70.2-75.5 \%$, respectively. (Table 4B).

\section{DISCUSSION}

Vascular injury sustained during PCI and stent implantation results in a complex inflammatory and reparative process. The acute vascular reaction is characterized by early deposition of platelets and fibrin. Activated platelets attach to circulating leukocytes (neutrophils and monocytes) at the injured surface. Over weeks, acute inflammatory cells are replaced by chronic inflammatory cells (macrophages and giant cells). In addition to this inflammatory response, platelet- and leukocyte-related growth factors drive further VSMC proliferation and migration from the media to the nascent neointima and subsequent extracellular matrix formation. Two weeks following stent

Table 4A. Cut-off values of MMP-3. LCAT and ApoE for ISR prediction.

\begin{tabular}{|c|c|c|c|c|c|c|}
\hline & \multicolumn{2}{|c|}{ MMP-9 } & \multicolumn{2}{|c|}{ LCAT } & \multicolumn{2}{|c|}{ ApoE } \\
\hline AUC & \multicolumn{2}{|c|}{0.715} & \multicolumn{2}{|c|}{0.771} & \multicolumn{2}{|c|}{0.775} \\
\hline Cut-off value & \multicolumn{2}{|c|}{$\geq 64.8(\mathrm{ng} / \mathrm{mL})$} & \multicolumn{2}{|c|}{$\leq 42.9(\mathrm{ug} / \mathrm{mL})$} & \multicolumn{2}{|c|}{$\leq 21.2(\mathrm{ug} / \mathrm{mL})$} \\
\hline Sensitivity & $65.8 \%$ & $56.2 \%-74.5 \%$ & $69.4 \%$ & $60.2 \%-78.2 \%$ & $67.7 \%$ & $57.7 \%-76.6 \%$ \\
\hline Specificity & $65.8 \%$ & $56.2 \%-74.5 \%$ & $72.0 \%$ & $62.5 \%-80.0 \%$ & $69.2 \%$ & $59.4 \%-77.9 \%$ \\
\hline PPV & $65.8 \%$ & $56.2 \%-74.5 \%$ & $71.7 \%$ & $62.1 \%-80.0 \%$ & $68.3 \%$ & $58.3 \%-77.2 \%$ \\
\hline NPV & $65.8 \%$ & $56.2 \%-74.5 \%$ & $70.0 \%$ & $60.5 \%-78.4 \%$ & $68.6 \%$ & $58.8 \%-77.3 \%$ \\
\hline False positivite & $34.2 \%$ & $25.5 \%-43.8 \%$ & $28.0 \%$ & $19.8 \%-37.6 \%$ & $30.8 \%$ & $22.1 \%-40.6 \%$ \\
\hline False negativite & $34.2 \%$ & $25.5 \%-43.8 \%$ & $30.3 \%$ & $21.8 \%-39.8 \%$ & $32.4 \%$ & $23.4 \%-42.3 \%$ \\
\hline
\end{tabular}

Table 4B. Cut-off values of indexed parametrs for ISR prediction.

\begin{tabular}{|c|c|c|c|c|c|c|}
\hline & \multicolumn{2}{|c|}{$\mathrm{LCAT}+\mathrm{ApoE}_{\text {index }}$} & \multicolumn{2}{|c|}{$\mathrm{LCAT}+\mathrm{ApoE}_{\text {index }}$} & \multicolumn{2}{|c|}{$\mathrm{ApoE}+\mathrm{ApoD}_{\text {index }}$} \\
\hline $\mathrm{AUC}$ & \multirow{2}{*}{\multicolumn{2}{|c|}{$\begin{array}{c}0.823 \\
\leq 65.4(\mathrm{ug} / \mathrm{mL})\end{array}$}} & \multirow{2}{*}{\multicolumn{2}{|c|}{$\begin{array}{c}0.823 \\
\leq 66.2(\mathrm{ug} / \mathrm{mL})\end{array}$}} & \multicolumn{2}{|c|}{0.783} \\
\hline Cut-off value & & & & & & $(\mathrm{ug} / \mathrm{mL})$ \\
\hline Sensitivity & $76.0 \%$ & $66.4 \%-84.0 \%$ & $77.0 \%$ & $67.5 \%-84.8 \%$ & $75.5 \%$ & $66.0 \%-83.5 \%$ \\
\hline Specificity & $75.5 \%$ & $66.0 \%-83.5 \%$ & $74.5 \%$ & $64.9 \%-82.6 \%$ & $70.2 \%$ & $60.4 \%-78.8 \%$ \\
\hline PPV & $75.3 \%$ & $65.7 \%-83.3 \%$ & $74.8 \%$ & $65.2 \%-82.8 \%$ & $71.3 \%$ & $61.8 \%-79.6 \%$ \\
\hline NPV & $76.2 \%$ & $66.7 \%-84.1 \%$ & $76.8 \%$ & $67.2 \%-84.7 \%$ & $74.5 \%$ & $65.7 \%-82.8 \%$ \\
\hline False positivite & $24.5 \%$ & $16.5 \%-34.0 \%$ & $25.5 \%$ & $17.4 \%-35.1 \%$ & $29.8 \%$ & $21.2 \%-39.6 \%$ \\
\hline False negativite & $24.0 \%$ & $16.0 \%-33.6 \%$ & $23.0 \%$ & $15.2 \%-32.5 \%$ & $24.5 \%$ & $16.5 \%-34.0 \%$ \\
\hline
\end{tabular}


implantation, a complete neointimal layer, composed of VSMCs and a proteoglycan-rich extracellular matrix, can be observed above stent struts ${ }^{1,2}$. Excessive VSMC proliferation and extracellular matrix formation lead to neointimal hyperplasia, which represents the major pathophysiologic mechanism of ISR. Peak restenosis is observed at 3-6 months and remains relatively stable beyond 1 year ${ }^{1,2}$.

We have used the MS-CT coronarography in order to exclude ISR in the control group especially due to its noninvasivness. 64- and more -slice MS-CT have been shown to permit the detection of coronary artery stenoses in the native coronary arteries with sensitivities and specificities up to $99 \%$. Owing to artefacts caused by metal, the visualization of the lumen within coronary artery stents by MS-CT is more challenging than the assessment of the native coronary arteries ${ }^{5}$. Sun Z. and Kumbahani DJ. evaluated the benefit of MS-CT in the detection of the coronary in-stent restenoses in their metaanalyses (nearly 1400 and 1500 evaluated stents). In these metaanalyses the sensitivity and specificity of MS-CT in detection of ISR achieved $90 \%$ and $91 \%$, with positive and negative predictive value of $68 \%$ and $98 \%$, respectively ${ }^{6,7}$.

Matrix metalloproteinases (MMPs) comprise a family of zinc-dependent proteolytic enzymes that degrade extracellular matrix (ECM) components and which facilitate the proliferation and migration of endothelial and vascular smooth muscle cells (VSMC).

MMPs are produced by various cell types, including endothelial and smooth muscle cells, and MMP activity is regulated by the naturally occurring molecules termed "tissue inhibitors of matrix metalloproteinases" (TIMPs).

MMP activity plays a role in the progression of ath erosclerotic lesions, arterial remodeling, and myocardial repair following infarction ${ }^{8}$. A mechanical injury induces expression of matrix metalloproteinases (MMP), and there is some evidence that they may play a role in the pathogenesis of ISR ( ref. $^{9-12}$ ).

We found that increased levels of MMP-3 and 9 were associated with a significant increase in ISR risk. The MMP-9 cut-off value for ISR risk prediction was determined to be $\geq 64.8 \mathrm{ng} / \mathrm{mL}$. In contrast, we did not find any correlation between MMP-2 and ISR incidence.

Leucocyte myeloperoxidase (MPO) is a member of the family of heme peroxidase enzymes. MPO is expressed in neutrophils and monocytes during inflammation, and it generates reactive oxygen species that can oxidatively modify lipids and proteins ${ }^{13}$. MPO has been found to be a component of human atherosclerotic lesions. MPO oxidizes low-density lipoprotein (LDL) into an atherogenic form (ox-LDL) and can contribute to endothelial dysfunction by decreasing nitric oxide (NO) bioavailability. Furthermore, MPO-generated hypochlorous acid can lead to matrix metalloproteinase (MMP) activation in the artery wall ${ }^{14}$. MPO levels were found to be significantly elevated in coronary artery disease (CAD) patients ${ }^{15}$, and it were associated with the progression from stable CAD to acute coronary syndromes (ACS) (ref. ${ }^{16}$ ). In our cohort, we found that increased plasma MPO was significantly associated with increased risk of ISR.

Nitric oxide (NO) plays a key role in vascular homeostasis. It is produced from L-arginine by nitric oxide synthase (NOS) and released from intact endothelium ${ }^{17}$. NO provides basal tonus by relaxing the vascular smooth muscle, and it inhibits the adhesion of leukocytes as well as the adhesion, activation, and aggregation of thrombocytes. Additionally, NO inhibits the migration and proliferation of smooth muscle cells, thereby it also inhibits intimal hyperplasia after vascular injury ${ }^{18,19}$.

Asymmetric dimethylarginine (ADMA) is an endogenous competitive inhibitor of endothelial nitric oxide synthase (eNOS). ADMA can lead to increased vascular tone and can accelerate the progression of atherosclerosis. Furthermore, by increasing VSMC proliferation and extracellular matrix formation, it may also influence the process of ISR (ref. ${ }^{20,21}$ ).

Previous reports showed an association between ISR and vasodilator dysfunction in forearm resistance vessels $^{18,21,22}$, and several small studies have reported an association between ISR and increased plasma ADMA levels ${ }^{17,18,20}$. In contrast to the previous studies, we did not find a link between increased ADMA and the risk of ISR. Conversely, we observed that increased levels of ADMA were associated with a significant reduction of the ISR risk.

Apolipoprotein E (ApoE) is a small apolipoprotein which serves as a ligand to the low-density lipoprotein (LDL) receptor (LDLR) and the LDL receptor-related protein-1 (LRP1), and plays an essential role in metabolism by promoting cellular uptake of lipoproteins ${ }^{23}$.

ApoE also inhibits platelet derivated growth factor (PDGF)-induced SMC migration and proliferation and limits neointimal hyperplasia after arterial injury ${ }^{24}$. ApoE deficiency has been associated with increased neointima formation after vessel wall injury ${ }^{25}$. In an ApoE-knockout mouse model, the in-stent neointimal area or the intimato-media (I/M) ratio was greater compared with wild-type mice $^{25}$. This effect of ApoE is independent from its lipidlowering effect ${ }^{23}$.

Although ApoE could play a protective role against injury-induced restenosis in animal models, the relationship between ApoE and ISR has not previously been studied in the general human population. We found that increased levels of ApoE were associated with a significant reduction in the risk of ISR and, moreover, that ApoE abundance represents one of the most important predictive factors associated with ISR protection.The ApoE cut-off value for ISR risk prediction was determined to be $\leq 21.2$ $\mathrm{ug} / \mathrm{mL}$.

Apolipoprotein D (ApoD) is an atypical apolipoprotein. In contrast to other apolipoproteins, ApoD is structurally similar to the lipocalins, which comprise a diverse class of lipid-binding proteins.ApoD has been shown 
to be a multi-ligand binding protein that is capable of transporting small hydrophobic molecules such as arachidonic acid, steroid hormones, and cholesterol. Circulating ApoD is predominantly found in HDL; however, ApoD is expressed widely in mammalian tissues. Human ApoD deposition is also detectable in atherosclerotic lesions ${ }^{26}$.

Several animal model studies suggest that intracellular accumulation of ApoD is able to selectively inhibit PDGF-induced VSMC proliferation and migration. This function of ApoD might thereby modulate the pathophysiology of vascular disease ${ }^{27}$. In our cohort, increased ApoD abundance was associated with a significant reduction in the risk of ISR.

Lipoprotein (a) (Lp[a]) is a large low-density lipoprotein (LDL)-like particle with the molecular structure of $\mathrm{Lp}$ (a) similar to that of plasminogen, and it may therefore have potential to reduce fibrinolysis by inhibiting the production of plasmin. After stent implantation, Lp(a) accumulates in the vessel walls at the sites of injury, where it also reduces activation of transforming growth factor-beta (TGF-beta), which is an inhibitor of SMC proliferation and migration ${ }^{28,29}$.

There is controversy regarding the relationship between baseline plasma $\mathrm{Lp}$ (a) levels and restenosis after coronary artery stenting ${ }^{29}$. A meta-analysis of 9 cohort studies found a significant association between baseline plasma Lp(a) levels and the risk of ISR, particularly in studies employing drug-eluting stent (DES) and in Asian populations but no statistical association was detected in studies with bare metal stents (BMS) or in Caucasian populations ${ }^{29}$. We also did not find any significant correlation between plasma levels of Lp (a) and ISR risk the Central European population.

High density lipoprotein (HDL) is a lipoprotein complex of heterogeneous size and density, with several different subpopulations. HDL also serves as a carrier for a number of enzymes that contribute to the remodeling of plasma lipoproteins. Many of these enzymes, such as lecithin-cholesterol acyl transferase (LCAT), affect the composition of circulating HDLor specific biologic activities associated with HDL (ref. ${ }^{30,31}$ ). The antiatherosclerotic effect of HDL comes from its contribution to reverse cholesterol transport (RCT). HDL has multiple additional biological functions, and it plays a complex role in the regulation of vascular activity. It may modulate endothelial function, platelet activation and thrombosis, inflammatory cytokine secretion, formation of foam cells, and SMC proliferation in the intima, resulting in the inhibition of neointimal hyperplasia ${ }^{31}$. HDL may also stimulate the differentiation of endothelial progenitor cells (EPCs) or the migration of endothelial cells from the adjacent healthy endothelium, and could thereby enhance endothelium repair after vascular injury, including stent re-endothelialization. It can also restore the activity of impaired endothelial nitric oxide synthase (eNOS). HDL may inhibit adhesion and activation of platelets and infiltration of inflammatory cells after vascular injury, which leads to decreasing of SMCs proliferation ${ }^{30}$.
Several studies have investigated possible relationships between HDL and ISR, with conflicting results, but in the meta-analysis by Heu no significant association was found overall between baseline HDL cholesterol (HDL-C) levels and ISR risk ${ }^{32}$.

Lecitin-cholesterol acyltransferase (LCAT) is a key enzyme acting in HDL metabolism. It mediates the esterification of free cholesterol that is then taken up from cells in peripheral tissue by the HDL particles. LCAT also plays an important role in the maturation of HDL, the esterification of cholesterol transforms the discoidal nascent HDL into spherical mature HDL particles ${ }^{33,34}$. Therefore, LCAT potentially influences not only RCT but also the other biological functions of HDL. Thus, LCAT is expected to contribute to the antioxidant and anti-inflammatory properties of HDL. Many studies have investigated the role of LCAT in atherosclerosis in animal models and humans; however, with conflicting results $^{34,35}$.

In our study, increased LCAT was associated with a significant reduction in the risk of ISR; it represents one of the most important protective factors observed. The LCAT cut-off value for ISR risk prediction was determined to be $\leq 42.9 \mathrm{ug} / \mathrm{mL}$. Given that there was no significant difference observed in the level of HDL cholesterol in plasma in the ISR group and the control group, this relationship may be explained by the effect of LCAT on maturation of HDL particles, which may be related to their different biological functions. Further research in this area will be necessary to elucidate the mechanisms responsible.

We suppose that screening of these biochemical parameters might be helpful in patients with an increased risk of ISR in whom routine implantation of drug eluting stents with long term dual antiaggregation therapy should be avoided, e.g. the patients with an increased bleeding risk or a need for urgent non-cardiac surgery.

\section{Limitations}

Several limitations of our study need to be mentioned. This study could be limited by its observational nature and a relatively small sample size. Nevertheless, we believe that selection bias did not play a major role, because both patient 's cohorts did not differ with respect to main baseline parameters. As non-invasive MS-CT coronarography was used in control group to confirm the stents patency, it is not possible to avoid completely potential false negatives findings. However, in previous studies, MSCT coronarography revealed sufficient accuracy for ISR detection $^{6,7}$.

\section{CONCLUSIONS}

Our study indicates that an increase in MMP-3, MMP-9, or MPO in plasma is associated with a significant increase in the risk of ISR after bare metal stent implantation. On the other hand, an increase in plasma 
ApoE, ApoD, or LCAT in plasma is associated with a significant reduction in the risk of ISR. In contrast to the results of other studies, increased ADMA in serum was also associated with a reduced risk of ISR. The most predictive parameters with respect to ISR were ApoE and LCAT abundance. No correlation was found between the abundance of MMP-2 and Lp (a) in plasma and the occurrence of ISR.

Acknowledgement: This article was co-supported by the project of European Social Fund and the State Budget of the Czech Republic, registration number CZ.1.07/2.3.00/20.0040 and by Ministry of Health, Czech Republic- conceptual development of research organization- FNOs/2013.

Author contributions: LP: study design; PP, LF, PK: literature search; LP, PK: data collection; PK, MK, PP, LF: data analysis; JZ: statistical analysis; LP: data interpretation; All authors: manuscript revision.

Conflict of interest statement: The authors state that there are no conflicts of interest regarding the publication of this article.

\section{REFERENCES}

1. Kim MS, Dean LS. In-Stent Restenosis. Cardiovasc Ther 2011;29:190-8

2. Byrne RA, Joner M, Massberg S, Kastrati A. Restenosis in bare metal and drug-eluting stents. In: Escaned J, Serruys PW, editors. Coronary stenosis, imaging, structure anf physiolohy. $1^{\text {st }}$ ed. Toulouse, France: Europa Edition; 2010.p.475-96.

3. Sun Z, Davidson R, Lin CH. Multi-detector row CT angiography in the assessment of coronary in-stent restenosis: A systematic review. Eur J Radiol 2009;69:489-95.

4. Kusnierova P, Vsiansky F, Pleva L, Plevova P, Svagera Z. [Estimation of reference intervals of biochemical markers predicting the risk of in-stent restenosis]. (Abstract) Clin Bioch Metab 2013;3:173.

5. Rixe J, Achenbach S, Ropers D, Baum U, Kuettner A, Ropers U, Bautz W, Daniel WG, Anders K. Assessment of coronary artery stent restenosis by 64-slice multi-detector computed tomography. Eur Heart J 2006;27:2567-72.

6. Sun Z, Marzouq A, Almutairi D. Diagnostic accuracy of 64 multislice CT angiography in the assessment of coronary in-stent restenosis: A meta-analysis. Eur J Radiol 73;2010:266-73.

7. Kumbhani DJ, Ingelmo CP, Schoenhagen P, Curtin RJ, Flamm SD, Desai MY. Meta-analysis of diagnostic efficacy of 64-slice Computed Tomography in the evaluation of coronary in-stent restenosis. Am J Cardiol 2009;103:1675-81.

8. Chen Q, Jin M, Yang F, Zhu J, Xiao Q, Zhang L. Matrix Metalloproteinases: Inflammatory Regulators of Cell Behaviors in Vascular Formation and Remodeling. Mediat Inflamm 2013;Article ID 928315 http://dx.doi.org/10.1155/2013/928315

9. Ge J, Shen C, Liang C, Chen L, Qian J, Chen H. Elevated matrix metalloproteinase expression after stent implantation is associated with restenosis. Int J Cardiol 2006;112:85-90.

10. Jones GT, Tarr GP, Phillips LV, Wilkins GT, van Rija AM, Williams MJA Active matrix metalloproteinases 3 and 9 are independently associated with coronary artery in-stent restenosis. Atherosclerosis 2009;207:603-7.

11. Katsaros KM, Kastl SP, Zorn G, Maurer G, Wojta J, Huber K, Christ G, Speidl WS. Increased Restenosis Rate After Implantation of DrugEluting Stents in Patients With Elevated Serum Activity of Matrix Metalloproteinase-2 and -9. J Am Coll Cardiol Intv 2010;3:90-7.

12. Tarr GP, Williams GT, Wilkins VHT, Chenb LV, Phillips AM, van Rij AM, Jones GT. Intra-Individual Changes of Active Matrix Metalloproteinase-9 Are Associated with Clinical In-Stent Restenosis of Bare Metal Stents. Cardiology 2013;124:28-35.

13. Dominguez-Rodriguez A, Abreu-Gonzalez P. Current role of myelo- peroxidase in routine clinical practice. Expert Rev Cardiovasc Ther 2011;9(2):223-30.

14. Ikitimur B, Karadag B. Role of myeloperoxidase in cardiology. Future Cardiol 2010;6(5):693-702.

15. Heslop CL, Frohlich JJ, Hill JS. Myeloperoxidase and C-reactive protein have combined utility for long-term prediction of cardiovascular mortality after coronary angiography. J Am Coll Cardiol 2010;55(11):1102-9.

16. Ndrepepa G, Braun S, Mehilli J, von Beckerath N, Schömig A, Kastrati A. Myeloperoxidase level in patients with stable coronary artery disease and acute coronary syndromes. Eur J Clin Invest 2008;38(2):906.

17. Bal UA, Yildirir A, Aydinalp A, Kaynar G, Kanyilmaz S, Murat K, Müderrisoglu IH. Could plasma asymmetric dimethylarginine level be a novel predictor beyond the classic predictors of stent restenosis? Anadolu Kardiyol Derg 2014;14:491-7.

18. Kitta Y, Nakamura T, Kodama Y, Takano H, Umetani K, Fujioka D, Saito Y, Kawabata K, Obata J, Ichigi Y, Mende A, Kugiyama K. Endothelial Vasomotor Dysfunction in the Brachial Artery Is Associated With Late In-Stent Coronary Restenosis. JACC 2005;46(4):648-55.

19. Ari $H$, Ari S, Erdogan $E$, Tiryakioglu $O$, Ustundag $Y$, Huysal $K$, Koca $V$ Bozat T. A novel predictor of restenosis and adverse cardiac events: asymmetric dimethylarginine. Heart Vessels 2010;25:19-26.

20. Derkacz A, Protasiewicz M, Poreba R, Doroszko A, Poreba M. Plasma asymmetric dimethylarginine predicts restenosis after coronary angioplasty. Arch Med Sci 2011;7:444-8.

21. Mizia-Stec K, Gasior Z, Haberka M, Mizia M, Chmiel A, Janowska J, Holecki M, Mielczarek M, Zahorska-Markiewicz B. In-stent coronary restenosis. but not the type of stent. is associated with impaired endothelial-dependent vasodilatation. Kardiol Pol 2009;67:9-17.

22. Thanyasiri P, Kathir K, Celermajer DS, Adams MR. Endothelial dysfunction and restenosis following percutaneous coronary intervention. Int J Cardiol 2007; 119:362-7.

23. Mendivil CO, Rimm EB, Furtado J, Sacks FM. Apolipoprotein E in VLDL and LDL With Apolipoprotein C-III is Associated With a Lower Risk of Coronary Heart Disease. J Am Heart Assoc 2013;2:e130

24. Koch W, Mehilli J, Pfeufer A, Schömig A, Kastrati A. Apolipoprotein E gene polymorphisms and thrombosis and restenosis after coronary artery stenting. J Lipid Res 2004;45:2221-6.

25. Ali ZA, Alp NJ, Lupton H, Arnold N, Bannister T, Hu Y. Increased InStent Stenosis in ApoE Knockout Mice. Insights from a Novel Mouse Model of Balloon Angioplasty and Stenting. Arterioscler Thromb Vasc Biol 2007;27:833-40.

26. Perdomo G, Dong HH. Apolipoprotein D in lipid metabolism and its functional implication in atherosclerosis and aging. Aging 2009;1:1727.

27. Sarjeant JM, Lawrie A, Kinnear C, Yablonsky S, Leung W, Massael H, Prichett W, Veinot JP, Rassart E, Rabinovitch M. Apolipoprotein D Inhibits Platelet-Derived Growth Factor-BB-Induced Vascular Smooth Muscle Cell Proliferated by Preventing Translocation of Phosphorylated Extracellular Signal Regulated Kinase 1/2 to the Nucleus. Arterioscler Thromb Vasc Biol 2003;23:2172-7.

28. Kamitani T, Taniguchi T, Miyai N, Kawasaki T, Kawasaki S, Sugihara $\mathrm{H}$. Association Between Plasma Lipoprotein(a) Concentration and Restenosis After Stent Implantation. Circ J 2005;69:644-9.

29. Qin S, Liu J, Jiang H, Huc B, Zhou Y, Olkkonen VM. Association between baseline lipoprotein (a) levels and restenosis after coronary stenting: Meta-analysis of 9 cohort studies. Atherosclerosis 2013;227:360-6

30. Yin K, Agrawal DK. High-Density Lipoprotein: A Novel Target for Antirestenosis Therapy. Clin Trans Sci 2014;3:1-12.

31. Le NA, Walter MF. Intrinsic enzymes of high-density lipoprotein. J Clin Lipidol 2007;1:20-30.

32. Heu WJ, Zhou Y, Liu JX, Liu J. Association of baseline high-density lipoprotein levels with restenosis after coronary stenting: a metaanalysis. Coronary Artery Dis 2013;24:386-391

33. Ono K. Current concept of reverse cholesterol transport and novel strategy for Atheroprotection. J Cardiol 2012;60:339-43.

34. Dominic SN. The role of lecithin:cholesterol acyltransferase in the modulation of cardiometabolic risks - A clinical update and emerging insights from animal models. Biochim Biophys Acta 2012;1821:654-9

35. van den Bogaard B, Holleboom AG, Duivenvoorden R, Hutten BA Kastelein JJP, Hovingh GK, Kuivenhoven JA, Stroes ESG, van den Born 
BJH. Patients with low HDL-cholesterol caused by mutations in LCAT have increased arterial stiffness. Atherosclerosis 2012;225:481-5.

Appendix 1. Reference intervals of normal values for ADMA. MMP-2. MMP-3. MMP-9 (ref. $\left.{ }^{4}\right)$.

\begin{tabular}{lcccc}
\hline Analyte (unit) & Number & Age $($ years $)$ & Gender & Reference interval $\left(2.5^{\text {th }}-97.5^{\text {th }}\right.$ centile values $)$ \\
\hline ADMA $(\mu \mathrm{mol} / \mathrm{L})$ & 120 & $34-61$ & Both & $0.227-0.877$ \\
MMP-2 $(\mu \mathrm{g} / \mathrm{L})$ & 62 & $50-62$ & Both & $160.8-550.2$ \\
MMP-3 $(\mu \mathrm{g} / \mathrm{L})$ & 22 & $48-61$ & F & $12.76-31.14$ \\
& 46 & & M & $18.03-42.04$ \\
MMP-9 $(\mu \mathrm{g} / \mathrm{L})$ & 58 & $19-61$ & F & $24.83-98.82$ \\
& 58 & & M & $33.37-108.67$ \\
\hline
\end{tabular}

M- male; F- female

Appendix 2. Biochemical parameters - categorized according to reference values.

\begin{tabular}{|c|c|c|c|}
\hline & In-stent restenosis & Controls & $P$ \\
\hline \multicolumn{4}{|c|}{ Matrix metalloproteinase-2 (MMP-2) } \\
\hline bellow & $48(44.0 \%)$ & $49(47.1 \%)$ & \multirow[t]{2}{*}{0.652} \\
\hline inside & $61(56.0 \%)$ & $55(52.9 \%)$ & \\
\hline \multicolumn{4}{|c|}{ Matrix metalloproteinase-3 (MMP-3) } \\
\hline bellow & $3(2.7 \%)$ & $5(4.5 \%)$ & \multirow{3}{*}{0.004} \\
\hline inside & $39(35.1 \%)$ & $62(55.9 \%)$ & \\
\hline above & $69(62.2 \%)$ & $44(39.6 \%)$ & \\
\hline \multicolumn{4}{|c|}{ Matrix metalloproteinase-9 (MMP-9) } \\
\hline bellow & $10(9.0 \%)$ & $37(33.3 \%)$ & \multirow{3}{*}{$<0.0001$} \\
\hline inside & $60(54.1 \%)$ & $59(53.2 \%)$ & \\
\hline above & $41(36.9 \%)$ & $15(13.5 \%)$ & \\
\hline \multicolumn{4}{|c|}{ Asymmetric dimethylarginine (ADMA) } \\
\hline bellow & $33(29.7 \%)$ & $18(16.7 \%)$ & \multirow[t]{2}{*}{0.022} \\
\hline inside & $78(70.3 \%)$ & $90(83.3 \%)$ & \\
\hline \multicolumn{4}{|c|}{ Lipoprotein (a) Lp(a) } \\
\hline bellow & $46(43.4 \%)$ & $40(37.0 \%)$ & \multirow[t]{2}{*}{0.343} \\
\hline inside & $60(56.6 \%)$ & $68(63.0 \%)$ & \\
\hline
\end{tabular}

$P$ - significance of chi-square test 
Appendix 3. ROC analysis.

\begin{tabular}{lcc}
\hline & AUC (Area Under the Curve) & $95 \%$ CI \\
\hline hs-CRP & 0.490 & $0.411-0.570$ \\
Lp(a) & 0.494 & $0.416-0.572$ \\
MMP-2 & 0.525 & $0.448-0.603$ \\
MMP-3 & 0.605 & $0.531-0.679$ \\
MMP-9 & 0.715 & $0.648-0.783$ \\
ADMA & 0.602 & $0.527-0.677$
\end{tabular}

\begin{tabular}{lcc}
\hline & AUC (Area Under the Curve) & $95 \%$ CI \\
\hline LCAT & 0.771 & $0.708-0.834$ \\
MPO & 0.635 & $0.562-0.709$ \\
ApoD & 0.675 & $0.602-0.747$ \\
ApoE & 0.755 & $0.689-0.820$ \\
LCAT + ApoE $_{\text {index }}$ & 0.823 & $0.765-0.881$ \\
ApoE + ApoD $_{\text {index }}$ & 0.783 & $0.720-0.846$ \\
\hline
\end{tabular}

Parameters with AUC $>0.7$ or $>0.75$ are suitable for the restenosis prediction

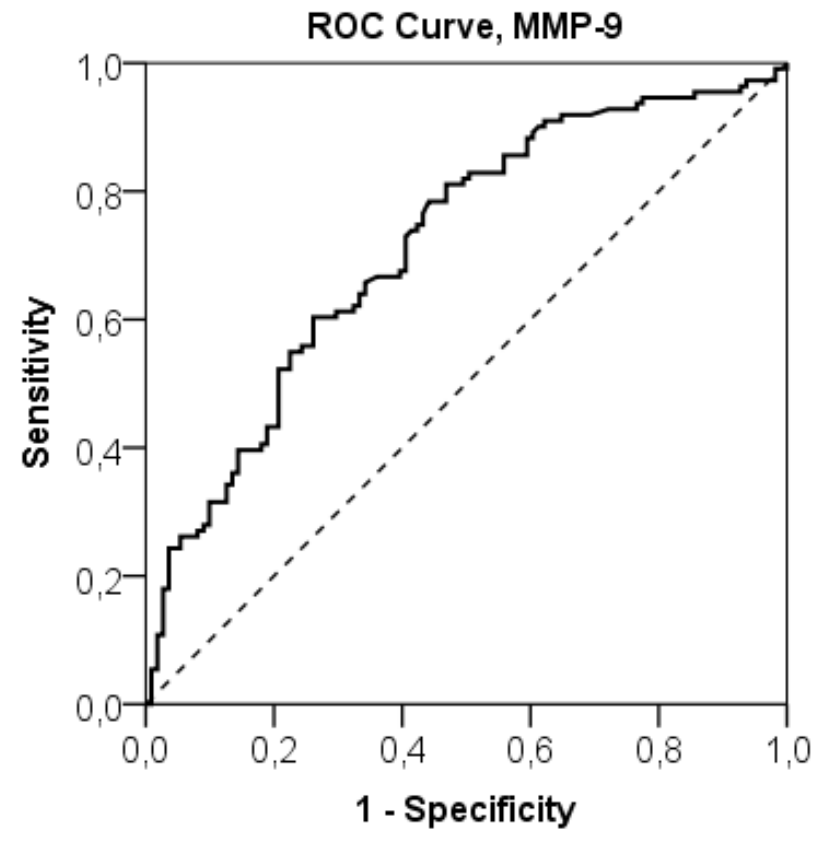

Diagonal segments are produced by ties.

RoC Curve, ApoE

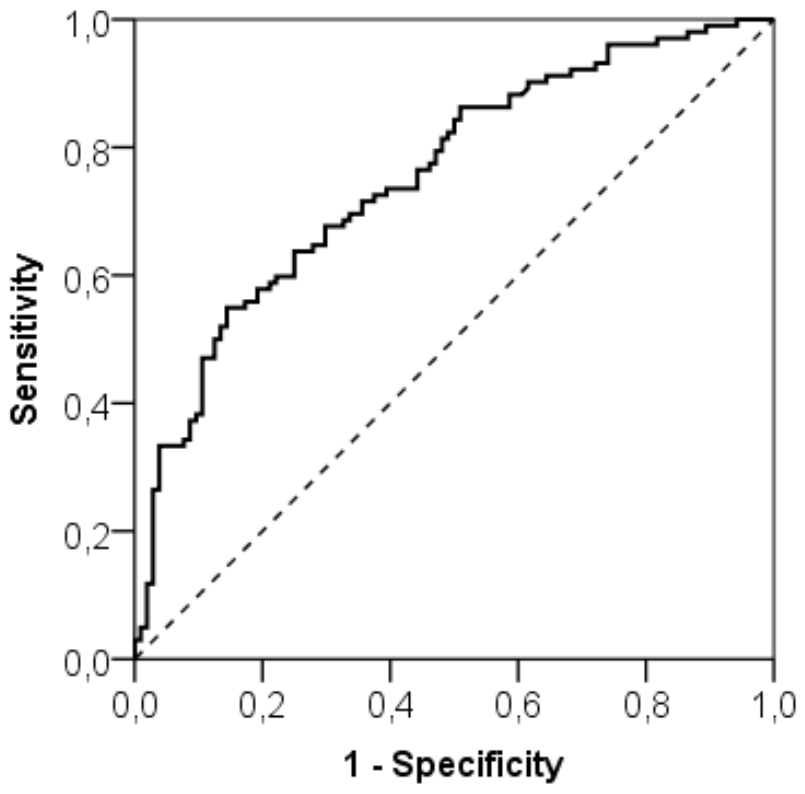

Diagonal segments are produced by ties.

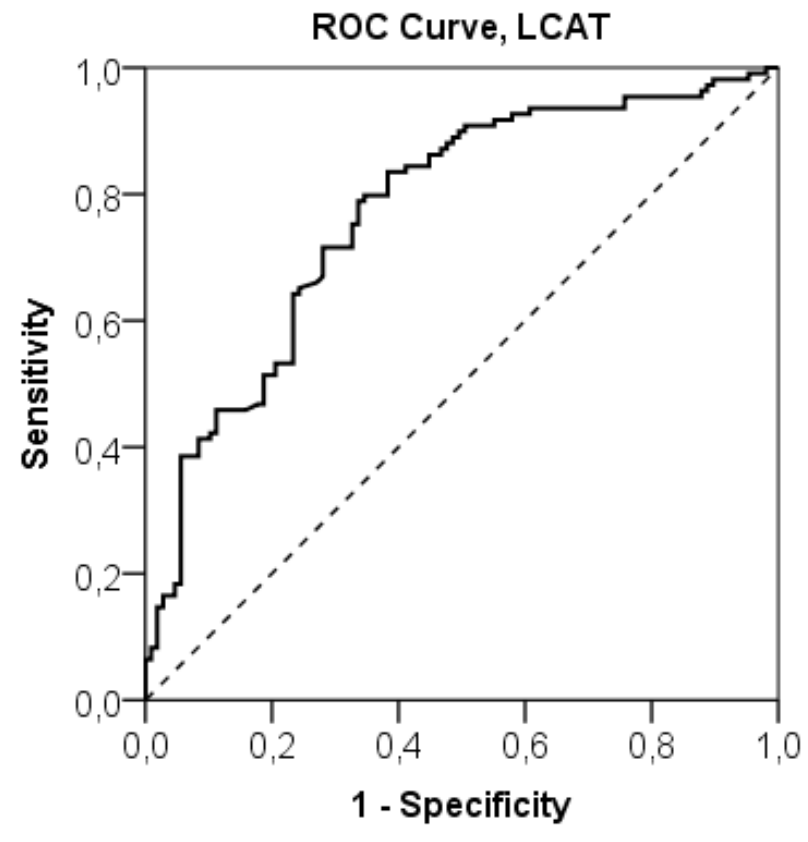

Diagonal segments are produced by ties.

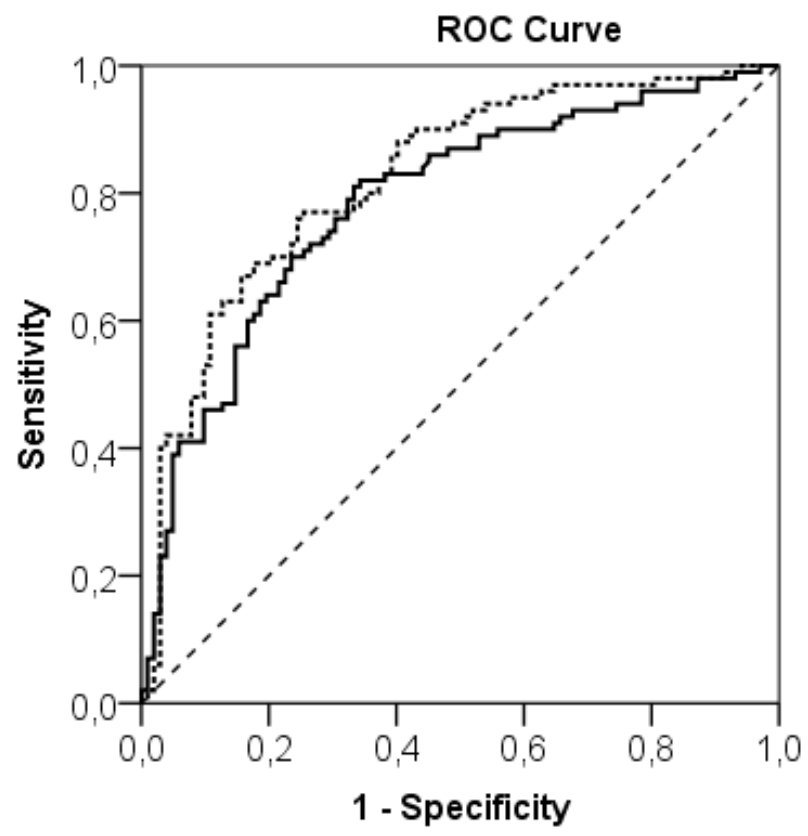

Diagonal segments are produced by ties.

Source of the Curve 